\title{
Le traitement motivé de l'information dans la prise de décision en groupe : le cas du paradigme des «profils cachés »
}

\author{
Claudia Toma ${ }^{1,2,3^{*}}$, Dimitri Vasiljevic ${ }^{4}$, Dominique Oberlé ${ }^{4}$, \\ Maria Augustinova ${ }^{5}$ et Fabrizio Butera ${ }^{6}$ \\ ${ }^{1}$ Université Catholique de Louvain, Louvain-la-Neuve, Belgique \\ ${ }^{2}$ Fonds National de la Recherche Scientifique \\ ${ }^{3}$ Université Pierre Mendès France, Grenoble, France \\ ${ }^{4}$ Université Paris Ouest Nanterre La Défense, France \\ ${ }^{5}$ CNRS et Université Blaise Pascal, Clermont-Ferrand, France \\ ${ }^{6}$ Université de Lausanne, Suisse
}

\section{RÉSUMÉ}

Quand des problèmes importants sont à résoudre, les groupes sont souvent utilisés pour prendre des décisions. Cet avantage accordé aux groupes repose sur l'hypothèse que les membres d'un groupe travaillent de manière coopérative et apportent des ressources supplémentaires lors de la prise de décision. Cet article reconsidère cette hypothèse classique de coopération et passe en revue les recherches effectuées dans le cadre du paradigme des «profils cachés » sous l'angle des enjeux compétitifs. À l'appui des études expérimentales récentes, cet article remet en question des résultats considérés comme classiques et offre des nouvelles perspectives de recherche quant au rôle des motivations coopératives et compétitives dans la prise de décision.

Motivated information processing in group decision making: the case of hidden profiles

\begin{abstract}
When complex problems are to be solved, groups are often used to make decisions. A popular reason for this is the assumption that group members work in a cooperative way and, by consequence, they make more informed decisions. This article reconsiders the classic cooperation assumption and reviews the literature on hidden-profiles from the
\end{abstract}

Correspondance : Claudia Toma, Université catholique de Louvain, Place du Cardinal Mercier 10, B-1348 Louvain-la-Neuve, Belgique. Email : claudia.toma@uclouvain.be

Ce travail a été soutenu par le programme «École et Sciences Cognitives 》 du Ministère français de l'Enseignement Supérieur et de la Recherche et du Fonds National belge de la Recherche Scientifique. 
perspective of competitive motivations. By using recent experimental evidence, this article casts doubt on some classic results and opens new perspectives of research with regard to the role of cooperative and competitive motivations on group decision-making.

L'étude de la qualité des décisions prises par les groupes est d'un réel intérêt pour les milieux organisationnels, éducationnels et politiques. En effet, quand des problèmes importants sont à résoudre, les groupes sont souvent utilisés pour donner des avis ou prendre des décisions. Par exemple, c'est aux membres d'un jury qu'il revient de déterminer la culpabilité d'un meurtrier présumé, aux états majors des directions des ressources humaines de discuter du candidat le plus apte à occuper un poste à responsabilité, aux conseils des prud'hommes de statuer sur les conflits au travail, aux staffs de médecins d'établir les diagnostics le plus appropriés.

L'usage de confier les décisions importantes à des groupes plutôt qu'à des individus s'est établi sur la supposition que la diversité des informations, des connaissances et des idées présente dans un groupe devrait assurer des décisions de qualité supérieure (Davis, 1996 ; Hastie, 1986 ; McGrath, 1984 ; Shaw, 1981 ; Steiner, 1972 ; Winquist \& Larson, 1998). La définition même du groupe repose sur l'idée de complémentarité informationnelle : les membres d'un groupe ont le potentiel de définir précisément le problème à résoudre, de générer les différentes solutions, de rassembler et évaluer les informations pertinentes, et sur ces bases, de choisir la meilleure alternative (Janis \& Mann, 1977 ; Moreland \& Levine, 1992). S’agit-il là d'une vision idéalisée du groupe ? Certains, depuis les fameux travaux de Janis (1972) sur le groupthink, se le demandent (Hart, 1998).

La supposition qu'en groupe s'opère une mise en commun des ressources informationnelles complémentaires, désignée souvent comme le «bonus d'assemblée » (Collins \& Guetzkow, 1964), repose clairement sur le postulat que les membres d'un groupe travaillent de manière coopérative lors de la prise de décision (Wittenbaum, Hollinghshead, \& Botero, 2004). Pourtant, bien que les groupes représentent en théorie un contexte idéal pour travailler en coopération, on doit se demander si la compétition n'y est pas également présente, et avec quels effets sur les décisions prises. Cette question est légitime dans la mesure où la compétition est omniprésente dans toutes les structures sociales, que ces dernières concernent la politique, les organisations ou encore l'éducation (Kasser, Cohn, Kanner, \& Ryan, 2007). C'est ainsi que par exemple, dans le système éducatif en France, la progression dans le programme académique est associée à une augmentation chez les étudiants de la reconnaissance de l'utilité de poursuivre des buts compétitifs (Darnon, Dompnier, Delmas, Pulfrey, \& Butera, 2009; Butera, Darnon, Buchs, \& Muller, 2006). 
Récemment, le modèle du traitement motivé de l'information dans le groupe (De Dreu, Nijstad, \& van Knippenberg, 2008) a montré l'intérêt de l'approche motivationnelle dans la prise de décision en groupe. À la lumière de ce modèle, notre article reconsidère le postulat selon lequel la prise de décision en groupe implique nécessairement la coopération. Plus spécifiquement, nous développons l'hypothèse originale que le bonus d'assemblée ne se réalise que rarement, puisque les membres du groupe sont motivés à la fois par la coopération et la compétition. Cela est dû au fait que les membres d'un groupe qui se réunissent afin d'atteindre un objectif commun (motivation coopérative) peuvent parfois avoir des objectifs personnels incompatibles avec le but commun (motivation compétitive) ou des objectifs communs et personnels à la fois (motivation mixte).

En nous appuyant sur des recherches expérimentales récentes, nous revisitons les travaux sur la prise de décision en groupe sous l'angle des motivations coopératives et compétitives. Pour cela, nous examinons d'abord les travaux classiques sur la prise de décision en groupe au regard de deux des principaux phénomènes inhérents à la discussion de groupe menant à une décision : 1. le rôle des positions individuelles préalables à la discussion (appelées aussi «préférences initiales »), 2. le partage de l'information durant la discussion.

\section{PRISE DE DÉCISION EN GROUPE : PARTAGER LES INFORMATIONS ET ÉCHANGER LES PRÉFÉRENCES}

La prise de décision en groupe est définie comme l'ensemble des processus impliqués dans le changement des préférences initiales des membres du groupe vers la position consensuelle de leur groupe (Kerr \& Tindale, 2004). Deux de ces processus apparaissent comme cruciaux vu leur influence sur la décision collective : ceux ayant trait aux préférences initiales des membres du groupe d'une part, et ceux concernant le partage des informations dont le groupe dispose d'autre part (Stasser \& Birchmeier, 2003).

Les premières recherches se sont presque exclusivement focalisées sur les préférences initiales des membres comme étant à l'origine des décisions de groupe (Lorge \& Salomon, 1955 ; Steiner, 1972). Les décisions étaient supposées émerger de la confrontation des préférences des uns et des autres, en fonction des normes du groupe (Kaplan \& Miller, 1987). Des modèles formels, plus prédictifs, ont ensuite été développés (par exemple, les schémas de décisions sociales, Davis, 1973 ou la séquence des interactions sociales, 
Stasser \& Davis, 1981). Ils stipulent que les groupes arrivent à un consensus en estimant statistiquement les préférences initiales selon des règles telles que « la majorité l'emporte », « l'unanimité l'emporte » ou « la vérité l'emporte » et précisent dans quelles conditions une règle plutôt qu'une autre est appliquée.

Bien que ces recherches occupent une place importante dans la littérature sur la prise de décision, les travaux les plus influents restent ceux qui se sont focalisés sur le partage de l'information (Brauner \& Scholl, 2000 ; Hinsz, Tindale, \& Volrath, 1997 ; Larson, Foster-Fishman, \& Keys 1994 ; Stasser \& Titus, 1985, 1987). La conception du groupe en tant qu'unité de traitement de l'information (Hinsz et al., 1997) est prédominante aujourd'hui. Elle repose sur l'idée que les groupes, autant que les individus, traitent l'information dans le but de résoudre des tâches intellectuelles et de prendre des décisions. Le partage de l'information est central à cette notion car il est vu comme l'activité cognitive prédominante dans les groupes (Tindale \& Kameda, 2000). C'est pourquoi, dans cette partie, après avoir présenté le paradigme des «profils cachés » (Stasser \& Titus, 1985, 1987) capital dans l'étude du partage des informations en groupe -, nous allons montrer comment les chercheurs ont pu mettre en lumière les modalités du partage d'informations ainsi que le rôle des préférences initiales.

\subsection{Le paradigme des «profils cachés» : un outil privilégié pour étudier la prise de décision en groupe}

Le nom du paradigme vient du fait qu'il distribue ingénieusement des informations à l'intérieur du groupe de manière à ce que les informations reçues individuellement et au préalable de la discussion «cachent » la décision optimale du groupe. Ce paradigme est un outil privilégié pour la prise de décision en groupe car il présente une forte validité écologique, dans la mesure où il correspond à des situations réelles dans lesquelles des individus divers (professionnels de la santé, ingénieurs, scientifiques...) possèdent des informations différentes sur un même problème à résoudre.

Suivons Stasser et Titus (1985), les promoteurs de ce paradigme, lorsqu'ils nous proposent d'imaginer la situation suivante : trois étudiants réunis en groupe ont pour consigne de désigner ensemble à partir des informations qui leur sont distribuées, le meilleur candidat, $\mathrm{A}$ ou $\mathrm{B}$, au conseil universitaire. La meilleure alternative est le candidat A dans la mesure où sept informations sont en sa faveur contre seulement quatre pour le candidat B. Or, dans la condition de «profil caché » (cf. Figure 1), une seule information en faveur de A est distribuée à tous les 
membres (information commune), les six autres sont réparties entre eux (informations uniques), de sorte que chacun n'a que trois informations en faveur de A (une commune et deux uniques) contre quatre en faveur de $\mathrm{B}$ (toutes communes), ce qui incite chacun à préférer $\mathrm{B}$. En conséquence, la décision optimale ne sera prise que si les membres du groupe partagent lors de la discussion les informations uniques dont ils disposent concernant A. L'idée de ce paradigme repose sur la mise en question de la logique de la théorie des arguments persuasifs (Burnstein \& Vinokur, 1977) selon laquelle l'information commune, étant redondante, devrait avoir peu d'impact durant la discussion, alors que l'information unique devrait être plus influente, car nouvelle pour ceux qui ne la détenaient pas au départ, et donc plus persuasive. Qu'en est-il effectivement?

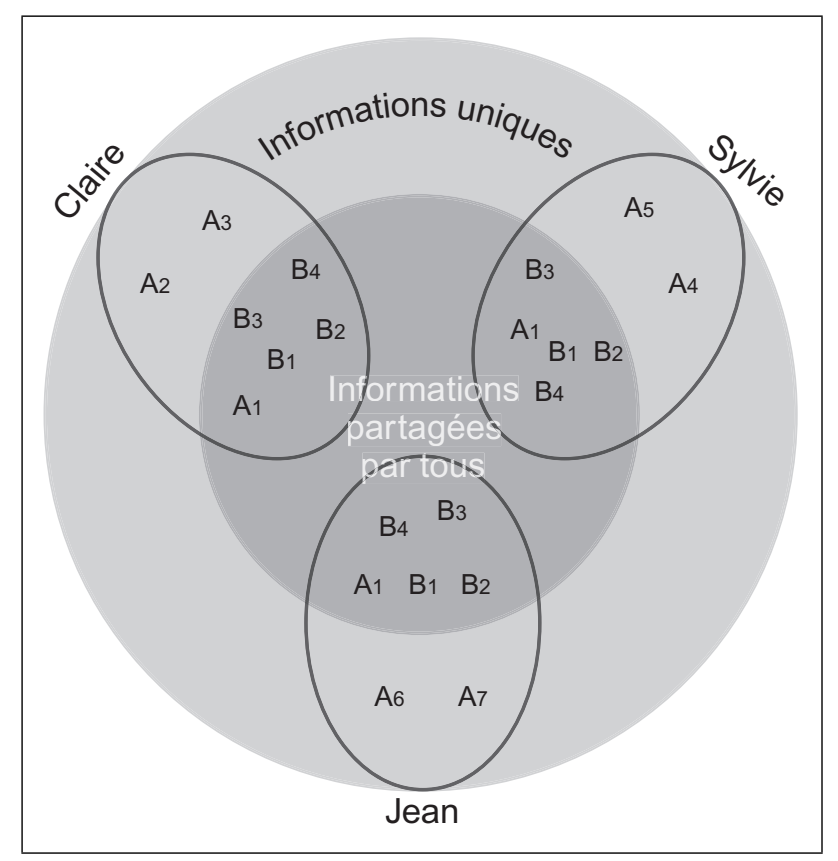

Figure 1. Distribution des informations au sein du groupe cachant l'alternative optimale utilisée par Stasser et Titus $(1985)^{1}$

\footnotetext{
${ }^{1}$ Figure tirée des archives personnelles de Gary Stasser, utilisée par Augustinova (2004). Les auteurs remercient Gary Stasser pour ce matériel.
} 


\subsection{Le partage et la répétition d'informations dans le paradigme des «profils cachés »}

Les résultats de l'étude princeps de Stasser et Titus (1985) montrent que ce n'est pas le cas. Dans la situation où toutes les informations sont communes, $83 \%$ des groupes sélectionnent le candidat A. En revanche, en situation de «profil caché », c'est-à-dire quand un certain nombre des informations sont uniques, seulement $18 \%$ des groupes le choisissent. Ces résultats ont été mainte fois répliqués : lors de la discussion de groupe, les membres discutent plus l'information commune que l'information unique ce qui amène le groupe à prendre de mauvaises décisions (Stasser \& Titus, 2003). Il est important de préciser que dans les recherches citées précédemment, les participants savent qu'ils ne disposent pas tous nécessairement des mêmes informations. Malgré cela, les informations uniques sont moins mentionnées que les informations communes lors des discussions collectives.

Pourtant, bien que l'information unique soit moins mentionnée que l'information commune, il est raisonnable de penser qu'une fois introduite, l'information unique est plus susceptible d'être répétée que l'information commune (Stasser, Taylor, \& Hanna, 1989). À cause de sa nouveauté, l'information unique devrait être plus persuasive et avoir plus d'impact sur les opinions que l'information commune (Burnstein \& Vinokur, 1977). En mettant cette hypothèse à l'épreuve des faits, Stasser et ses collègues (1989) obtiennent un effet inverse, notamment une plus grande répétition de l'information commune que de l'information unique.

Pour rendre compte de ces différents résultats, Stasser et Titus (1987) ont proposé que le partage d'informations en groupe soit régi par les mêmes lois probabilistes que le processus d'échantillonnage. Le modèle CIS (Collective Information Sampling model) qui en découle reprend l'idée de Steiner (1972) selon laquelle une information sera discutée à condition qu'au moins un membre du groupe la mentionne. Ainsi, la probabilité que le groupe discute une information est fonction du nombre de membres qui peuvent mentionner cette information et de la probabilité qu'un seul membre du groupe évoque l'information. Pour cette raison, l'information commune est susceptible d'être mentionnée par chaque membre du groupe ; l'information unique, en revanche, ne peut être mentionnée que par un seul individu. Stasser et ses collègues (1989) appliquent cette même logique probabiliste à la répétition des informations uniques. Ils proposent qu'une fois mentionnée, l'information unique n'est pas aussi facile à rappeler que l'information commune dans le sens où les membres du groupe ont déjà été exposés deux fois à l'information commune (une 
fois avant et une fois pendant la discussion) alors qu'ils n'ont été exposés qu'une seule fois à l'information unique (pendant la discussion) quand ce n'était pas eux qui en disposaient au départ.

En sus de ces explications strictement statistiques, Larson et Harmon (2007) suggèrent que les informations communes sont plus compréhensibles que les informations uniques parce qu'elles sont déjà connues au départ, et donc plus fiables. Alors que tous peuvent valider l'information commune, aucun membre du groupe ne peut valider l'information unique, hormis celui qui l'a mentionné la première fois. C'est pourquoi les membres du groupe évitent de la répéter (Parks \& Cowlin, 1996). L'hypothèse de «non-fiabilité » a été testée par Schittekatte et Van Hiel (1996). Ils montrent ainsi que l'information détenue par deux personnes dans un groupe de quatre est deux fois plus répétée que lorsqu'elle n'est détenue que par une seule personne. Cela confirme l'idée que la validité d'une information et la possibilité qu'elle soit répétée au sein du groupe augmentent avec le nombre de personnes qui la détiennent.

Pour résumer, les informations uniques pourtant indispensables pour la découverte de la décision optimale ont un double désavantage, comparées aux informations communes : elles sont non seulement moins susceptibles d'être mentionnées durant la discussion mais elles sont aussi moins susceptibles d'être répétées. En somme, ces différents résultats et les explications qui en sont données, tout comme leurs multiples réplications (pour des revues de question voir Stasser, 1999 ; Stasser \& Titus, 2003) suggèrent que la discussion collective est rarement centrée sur un examen systématique des informations dont le groupe dispose.

\subsection{L'échange des préférences initiales dans les profils cachés}

Le non-partage de l'information unique pendant la discussion est-il la seule cause des prises de décision sous-optimales dans le groupe ? La question se pose puisque dès 1983, dans leur travail sur la formation du verdict dans les jurys d'assise, Hastie, Penrod et Pennington avaient fait une distinction théoriquement importante entre prise de décision centrée sur la discussion des preuves (c'est-à-dire, les informations dont le groupe dispose) et prise de décision centrée sur la discussion des préférences individuelles concernant la condamnation (c'est-à-dire, les préférences individuelles formées avant la discussion de groupe). Or, il faudra attendre les travaux de Gigone et Hastie $(1993,1997)$ pour que soit mis en évidence un autre facteur déterminant dans la prise de décision : l'échange des préférences initiales des membres du groupe. 
Dans leur étude, des groupes de trois personnes doivent prédire la note obtenue par 32 étudiants à un cours d'introduction en psychologie. Avant la discussion, six informations sont distribuées de telle sorte que deux informations sont délivrées à tous les membres (informations communes), deux sont données à deux membres (informations partiellement partagées) et deux à un seul (informations uniques). Les résultats mettent en évidence que plus il y a de membres qui possèdent l'information avant la discussion, plus elle est discutée et plus elle a d'impact sur la décision finale. Toutefois, les résultats révèlent surtout que la distribution initiale de l'information et les préférences initiales qui en découlent influencent la décision du groupe même lorsque la discussion des informations a été optimale. Ainsi, selon ces auteurs, le partage et la discussion des informations n'auraient finalement qu'un impact limité sur la décision du groupe et ne permettraient que la négociation du poids de chaque jugement initial dans la décision finale. De ce fait, les décisions collectives résulteraient davantage de la combinaison des positions individuelles préalables à la discussion que du partage des informations reçues par chacun des participants. Il s'agit là d'une explication alternative au fait que les informations communes sont plus discutées que les informations uniques.

Plus radicalement encore, Greitemeyer et Schulz-Hardt (2003) proposent que la difficulté à découvrir les profils cachés s'explique en dehors des processus collectifs dysfonctionnels par des processus individuels opérant en amont et indépendamment de la discussion de groupe. Dans leur paradigme dit «sans discussion », ces auteurs demandent aux participants d'imaginer qu'ils font partie d'un groupe qui va devoir prendre une décision. Un scénario sous forme de profil caché leur est distribué une première fois individuellement. Ils ont ensuite l'occasion, toujours individuellement, de réviser leur opinion après avoir lu une discussion fictive qui fait apparaître l'intégralité des informations nécessaires à la découverte de la bonne décision. Pourtant, même dans cette situation idéale où ils disposent de toutes les informations, les participants échouent à la tâche et prennent une mauvaise décision. Ceci s'explique, selon les auteurs, par le fait que les individus, peu disposés à remettre en cause leurs préférences initiales, considèrent difficilement les informations inconsistantes avec celles-ci. En d'autres termes, ils sont victimes du «biais de confirmation » (Lewicka, 1989), également dénommé « effet d'exposition sélective à l'information » (Frey, 1986).

Enfin, récemment, Mojzisch et Schulz-Hardt (2010) ont montré que le simple fait d'être focalisé sur les préférences initiales des uns et des autres conduisait à une décision sous-optimale. Les participants de 
leur étude, s'imaginant participer à une prise de décision en groupe, recevaient individuellement des informations de manière à être orientés initialement vers une décision sous-optimale. Par la suite, certains étaient mis au courant des préférences des autres et recevaient par écrit les informations des autres membres du groupe. Au vu de ces nouvelles informations, les participants étaient invités à prendre une décision finale. Les résultats de cette étude montrent que les participants ayant été informés de la distribution des préférences initiales dans le groupe, ont été plus nombreux à prendre une mauvaise décision que ceux n'ayant pas reçu cette information. Autrement dit, cette étude et celle de Greitemeyer et Schulz-Hardt (2003) montrent que la découverte de la décision optimale n'est pas possible tant que les membres du groupe n'admettent pas de prendre en considération et de traiter de façon critique les informations qui vont à l'encontre de leurs préférences initiales.

\subsection{Le modèle des processus conjoints}

Comme on vient de le voir, selon les chercheurs et leur orientation théorique, l'incapacité des groupes à résoudre les profils cachés est à mettre sur le compte soit d'un partage de l'information défaillant (Larson et al, 1994 ; Larson, Christensen, Abbott, \& Franz., 1996 ; Larson \& Harmon, 2007 ; Parks \& Cowlin, 1995 ; Savadori, Van Swol, \& Sniezek, 2001 ; Stasser \& Birchmeier, 2003 ; Stasser \& Davis, 1981 ; Stasser \& Stewart, 1992 ; Stasser, Stewart, \& Wittenbaum, 1995 ; Stasser \& Titus, 1985), soit d'une centration sur les préférences initiales (Brodbeck, Kerschreiter, Mojzisch, Frey, \& Schulz-Hardt, 2002 ; Gigone \& Hastie, 1993, 1997 ; Greitemeyer \& Schulz-Hardt, 2003; Schulz-Hardt, Brodbeck, Mojzisch, Kerschreiter, \& Frey, 2006 ; Mojzisch \& Schulz-Hardt, 2010).

Cependant, à travers leur «modèle des processus conjoints » (dual process model), Winquist et Larson (1998) intègrent ces deux approches. Pour ces auteurs, en effet, l'impact des informations communes sur la prise de décision est médiatisé par les préférences initiales alors que l'impact des informations uniques est médiatisé par la discussion de groupe. Plus spécifiquement, les informations communes affectent la décision collective car elles déterminent les préférences initiales (Gigone \& Hastie, 1993) qu'elles soient ou non partagées durant la discussion. Ce biais est pourtant susceptible d'être éliminé durant la discussion en y intégrant les informations uniques. L'impact conjoint des préférences initiales et du partage d'informations est aujourd'hui reconnu dans des élaborations théoriques (Tindale, \& Kameda, 2000 ; Stasser \& Dietz-Uhler, 
2001 ; Tindale, Meisenhelder, Dykema-Engblade, \& Hogg, 2001) et travaux empiriques (Augustinova, 2008 ; Toma \& Butera, 2009).

\section{APPROCHE MOTIVATIONNELLE DE LA PRISE DE DÉCISION EN GROUPE}

Si les recherches présentées dans les sections précédentes représentent une avancée importante à la compréhension des processus impliqués dans les décisions de groupe, la plupart d'entre elles sont fondées sur le présupposé que les membres d'un groupe travaillent de manière coopérative (Wittenbaum et al., 2004). Ce postulat repose sur l'idée que si les membres d'un groupe sont réunis pour prendre une décision commune, ils vont nécessairement travailler de manière coopérative.

De plus, les recherches antérieures ont été réalisées dans le cadre d'une approche opératoire des groupes qui a comme objectif l'identification de facteurs stables qui contribuent à la prise d'une décision collective. Cette approche instruit davantage sur « comment les choses devraient se passer » plutôt que sur « comment elles se passent effectivement», avec une centration sur la tâche et non sur le groupe en tant que tel. La question est cependant de savoir si ce présupposé reflète réellement les situations de prise de décision en groupe. L'intérêt pour la dimension opératoire de la prise de décision a fait oublier l'existence de la dimension socio-motivationnelle qui renvoie aux membres du groupe, à leurs relations, à leurs buts et à leurs motivations. Or, ce qui se passe dans un groupe, les résultats auxquels il arrive, dérivent de la combinaison des deux sortes de phénomènes, ceux centrés sur la tâche et ceux relatifs aux membres (Darnon, Muller, Schrager, Pannuzzo, \& Butera, 2006).

Certes, la coopération paraît être le type de fonctionnement le mieux adapté à des tâches caractérisées par l'interdépendance des ressources comme le sont les «profils cachés ». Mais elle ne découle pas nécessairement des phénomènes de groupe centrés sur la tâche. En fait, et compte tenu de leurs enjeux identitaires et de leur besoin d'une valorisation d'eux-mêmes, les membres d'un groupe sont souvent guidés par des motivations mixtes : une motivation coopérative qui vise la réussite du groupe coexistant avec une motivation compétitive qui vise la réussite individuelle face aux autres membres du groupe (Davis, Laughlin, \& Komorita, 1976). Autrement dit, même si les recherches antérieures ont supposé que les décisions de groupe ont été prises de manière coopérative, elles reflètent probablement plus les motivations mixtes que les motivations purement coopératives des membres du groupe. 


\subsection{Le modèle du traitement motivé de l'information dans le groupe (MIP-G, De Dreu et al., 2008)}

Cette idée est également soutenue par le modèle du traitement motivé de l'information dans le groupe (MIP-G, De Dreu et al., 2008). Selon ce modèle « les théories et la recherche sur la prise de décision en groupe pourraient être enrichies par la prise en considération des motivations mixtes coopérative et compétitives qu'ont les individus qui travaillent en groupe » (p. 24). Les auteurs de ce modèle font remarquer que le présupposé de la coopération dans les recherches sur la prise de décision en groupe est inconsistant avec les travaux sur la formation des coalitions (Komorita \& Parks, 1995), ainsi qu'avec les travaux sur la négociation (Pruitt, 1998 ; De Dreu \& Carnevale, 2003), dans lesquels un présupposé de compétition est plus présent. Plusieurs motivations compétitives (le désir d'avoir un haut statut, de prouver sa compétence, ou d'être reconnu comme celui/celle qui amène le groupe à réussir) sont présentes dans les groupes et influencent les choix stratégiques et le traitement de l'information (Buchs, Pulfrey, Gabarrot, \& Butera, 2010 ; Darnon, Butera, \& Harackiewicz, 2007 ; De Dreu \& Carnevale, 2003 ; De Dreu, Beersma, Stroebe, \& Euwema, 2006 ; Steinel \& De Dreu, 2004). L'argument clé à l'origine de ce modèle est que toutes les situations de prise de décision en groupe impliquent l'interdépendance de ses membres (Buchs, Butera, \& Mugny, 2004), et que ces situations d'interdépendance sont susceptibles de générer des conflits entre les intérêts collectifs et les intérêts individuels (Kelley \& Thibaut, 1978), d'où l'existence de motivations mixtes, à la fois coopératives et compétitives.

\subsection{Les motivations mixtes: coopératives et compétitives}

Bien qu'une vaste littérature suggère que les individus diffèrent quant à leur orientation motivationnelle (par ex., McClintock \& Liebrand, 1988 ; Kelley \& Stahelski, 1970), le concept de motivation abordé dans ce chapitre se réfère à la motivation en tant que variable contextuelle. Nous défendons ici une conception directionnelle de la motivation comme étant dirigée par un but identifiable et induite régulièrement par un contexte spécifique et/ou un jeu de consignes qui permettent l'activation d'un but (Darnon et al., 2007 ; De Dreu \& McCusker, 1997 ; Chaiken, Giner-Sorolla, \& Chen, 1996 ; Webster \& Kruglanski, 1998). Cette manière de procéder est cohérente avec le constat selon lequel les buts des individus sont induits par les contextes dans lesquels ils évoluent (Wittenbaum et al., 2004). La théorie de la coopération et compétition (Deutsch, 1973 ; Johnson \& Johnson, 1989) suggère qu'en fonction du contexte, les membres d'un groupe peuvent 
percevoir leurs buts comme étant positivement liés ce qui amène à la coopération ou négativement liés ce qui amène à la compétition (voir aussi Tjosvold, 1986, 1990).

De plus, à l'intérieur d'une même situation groupale, on peut trouver en même temps des buts communs à tous les membres, et d'autres qui sont spécifiques à certains membres, ce qui implique que la coopération et la compétition peuvent coexister. C'est bien en effet ces motivations mixtes dont l'approche motivationnelle de De Dreu et al. (2008) révèle l'existence. Les motivations mixtes se référent aux situations dans lesquelles les individus sont face à un dilemme entre la volonté de coopérer pour le bien collectif et la possibilité d'entrer en compétition afin d'atteindre des objectifs personnels (Komorita \& Parks, 1995). Dans certaines situations de prise de décision, la coopération est prédominante, alors que dans d'autres, c'est la compétition qui l'est. Une situation devient coopérative ou compétitive en fonction des buts qui sont activés dans un contexte donné et en fonction de l'interdépendance perçue entre ces buts. Autrement dit, ce n'est pas parce que les membres d'un groupe ont des buts individuels qu'ils vont forcément être compétitifs. La compétition apparaît uniquement lorsqu'il y a incompatibilité entre les buts individuels et les buts collectifs.

Dans les sections suivantes, nous allons montrer l'impact des motivations mixtes en nous appuyant sur des études expérimentales qui ont manipulé les contextes induisant des buts coopératifs et compétitifs dans les situations de prise de décision de type profil caché.

\subsection{Le dilemme dans les profils cachés: partager ou garder pour soi l'information unique, quelle est la meilleure stratégie ?}

Les « profils cachés » (Stasser \& Titus, 1985, 1987) représentent le prototype des situations de prise de décision dans lesquels les membres du groupe sont interdépendants. Chacun possède une partie de l'information que les autres n'ont pas, et chacun a besoin des informations des autres pour une prise de décision optimale. Imaginez la situation suivante (l'exemple est tiré de Toma \& Butera, 2009) : Trois membres sont réunis en groupe pour décider qui parmi trois suspects potentiels a provoqué un accident de voiture. Tous les membres de ce groupe détiennent des informations communes liées aux suspects et aux conditions dans lesquelles l'accident a eu lieu. Par ailleurs, chaque personne dans le groupe a des informations que les autres ignorent et qui permettent d'identifier le coupable (informations uniques). $\mathrm{Au}$ vu des seules informations qui leur ont été distribuées, et avant qu'une 
discussion ne donne l'occasion de partager l'information unique, aucun des membres du groupe ne peut trouver la solution.

Dans cette situation, chacun a besoin des autres pour réussir. Chacun des membres du groupe a besoin des informations des autres pour trouver la solution du dilemme. Mais certains peuvent agir dans l'intérêt du groupe et mentionner les informations dont les autres ne bénéficient pas (l'information unique), alors que d'autres peuvent agir dans ce qu'ils croient être leur intérêt individuel et faire de la rétention de l'information unique. En gardant pour soi l'information unique, chacun de membres peut empêcher les autres de trouver la solution correcte, et s'assurer ainsi un avantage compétitif.

Ce n'est donc pas parce que les situations de type "profil caché » nécessitent un travail coopératif, que les membres du groupe sont disposés à être coopératifs ( $c f$. Wittenbaum et al. 2004). D’ailleurs, les études réalisées dans le cadre de ce paradigme (voir la partie 1) révèlent bien que les participants échouent à prendre la bonne décision (Larson et al., 1994 ; Stasser \& Titus, 1985, 1987). C'est donc qu'ils échouent à coopérer, alors même que la tâche requiert la coopération (Tindale \& Sheffey, 2002). Nous faisons alors l'hypothèse originale que l'absence de partage des informations uniques dans le paradigme du profil caché peut être expliquée par les motivations compétitives des membres du groupe. Cette hypothèse n'est pas sans rappeler l'approche de French et Raven (1959). Ces auteurs soutiennent que le contrôle de l'information est une base importante de pouvoir social. Celui qui contrôle l'accès à l'information critique empêche les autres de réussir et assure sa supériorité et son statut dominant dans le groupe. Cependant, dans une tâche caractérisée par l'interdépendance des ressources, la rétention d'informations si elle devient systématique risque d'être pénalisante in fine pour tous puisqu'elle rend impossible la découverte de la solution optimale. Les participants guidés par des motivations compétitives s'en rendent-ils compte, ou sont-ils aveuglés par ces motivations?

\subsection{L'impact de la compétition sur la prise de décision: partage et utilisation stratégique de l'information}

Pour le savoir, Toma et Butera (2009), dans une recherche qui contrastait expérimentalement des groupes induits à travailler en coopération vs en compétition, ont testé l'hypothèse d'une utilisation stratégique de l'information. La tâche proposée, de type profil caché, (voir Figure 2) avait été conçue pour tester le rôle respectif des préférences initiales et du partage 


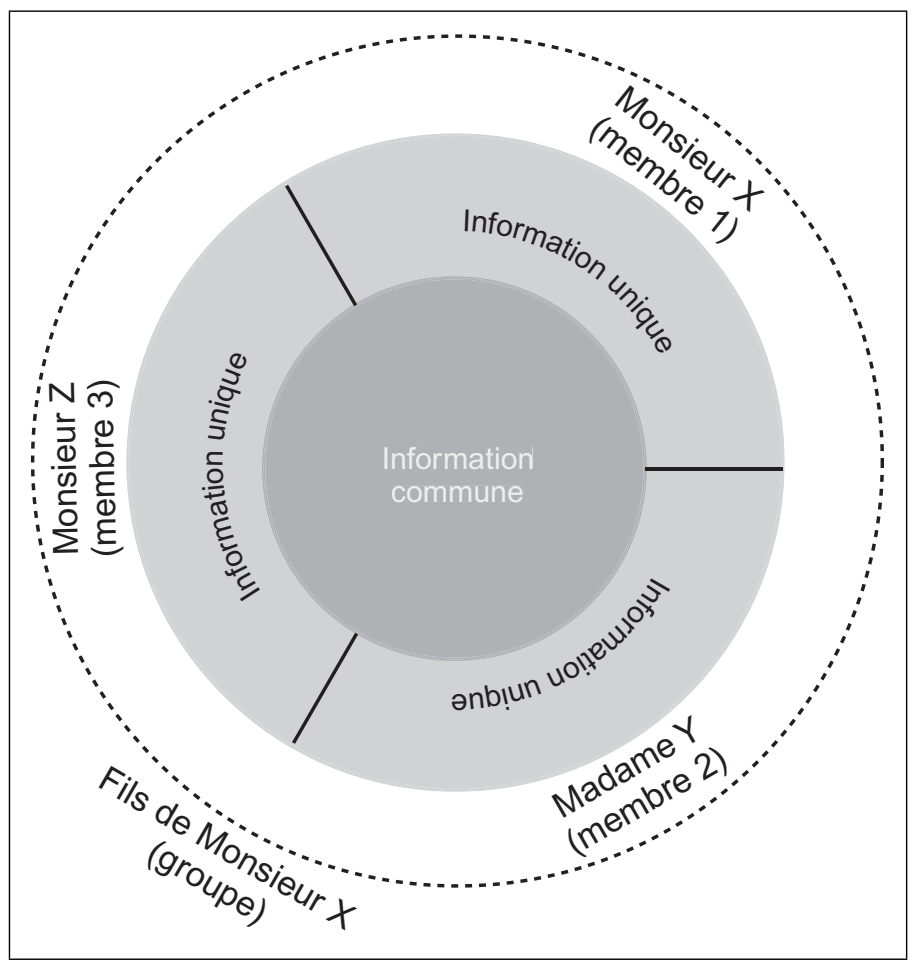

Figure 2. La distribution de l'information et les préférences initiales dans le paradigme « profil caché » (selon Toma \& Butera, 2009)

\section{d'informations sur la décision ${ }^{2}$. Elle consistait à discuter en groupe à partir des informations que chaque membre avait reçues, pour trouver parmi}

\footnotetext{
${ }^{2}$ Premièrement, la tâche était transparente afin de permettre aux membres du groupe d'identifier avec exactitude les informations uniques et les informations communes. Une étude pilote avait montré qu'en indiquant aux participants l'emplacement de deux types d'informations, ils étaient capables d'identifier avec précision quelles étaient les informations communes et quelles étaient les informations uniques. De plus, une autre étude pilote avait montré la capacité des participants à identifier la supériorité de valeur informationnelle de l'information unique comparée à celle de l'information commune pour résoudre la tâche. Comme la tâche permettait aux participants d'identifier l'emplacement et la valeur des informations, logiquement elle devait faciliter le partage de l'information critique pour la découverte du profil caché.

Deuxièmement, les informations uniques qui orientaient chaque participant vers des préférences sous-optimales, permettaient - si elles étaient partagées - l'infirmation de ces préférences initiales erronées. Par exemple l'information unique « la personne responsable est un homme » orientait celui qui la détenait vers le suspect « Monsieur X 》 mais infirmait la préférence initiale d'un autre membre qui par sa propre information unique avait été orientée sur « Madame Y 》. Trois études pilotes avaient mis en évidence qu’il était possible d'orienter les trois membres du groupe vers trois solutions différentes, alors qu'une quatrième étude pilote avait montré que par la prise en compte des informations uniques, il était possible de prendre la bonne décision.
} 
plusieurs suspects le véritable auteur d'un accident de voiture. En coopération, les participants étaient informés que l'objectif du groupe était de trouver le coupable de l'accident. En revanche, en compétition, les participants étaient informés que le groupe devait trouver le coupable de l'accident, mais qu'il était important d'être le premier à trouver la bonne solution.

Leur première étude met en évidence que les membres des groupes induits vers la compétition partagent moins d'informations uniques, sont plus réticents à infirmer leurs solutions initiales et prennent moins de décisions correctes que les membres des groupes induits vers la coopération.

Cependant, cette première étude n'éclaire guère sur les raisons sous-jacentes à la rétention des informations uniques en compétition, ni sur celles qui dans le même contexte rendent difficile l'infirmation des préférences initiales. Définie comme la réticence à se montrer vulnérable et sous le contrôle des autres, la méfiance est responsable de comportements de tricherie tels que la rétention de l'information (Steinel \& De Dreu, 2004). Les résultats d'une deuxième étude montrent effectivement que la méfiance est responsable de la rétention de l'information unique et de la réticence à infirmer les préférences initiales. Ils confirment en outre les conclusions d'études antérieures (Butera \& Mugny, 1995, 2001 ; De Dreu \& van Knippenberg, 2005) montrant qu'en compétition, la difficulté d'infirmer ses préférences initiales semble être déterminée par le besoin de protéger sa solution face à d'autres compétiteurs, alors que la pensée critique s'avère être plus présente dans les groupes en coopération (Postmes, Spears, \& Cihangir, 2001).

Ainsi, conformément au propos défendu dans cet article à partir du modèle du traitement motivé de l'information dans le groupe (De Dreu et al., 2008), cette recherche de Toma et Butera (2009) offre une nouvelle perspective quant à la manière de conceptualiser le traitement de l'information dans les groupes. Elle montre que lorsque les membres d'un groupe dépendent des informations des autres pour aboutir à une décision optimale, la tentation compétitive pousse à la rétention de l'information et à l'échec de la décision.

\section{DE L'OPTIMISATION DES DÉCISIONS DE GROUPE : LE RÔLE DES MOTIVATIONS COOPÉRATIVES ET COMPÉTITIVES}

$\mathrm{Vu}$ les entraves à de bonnes décisions de groupe, il n'est pas étonnant que de nombreux chercheurs aient étudié les facteurs susceptibles de 
surmonter ces obstacles. Cependant, l'influence positive ou facilitatrice de ces facteurs a été testée sous l'angle implicite de la coopération discuté précédemment (voir partie 2). Dans ce qui suit nous avançons que la prise en compte de motivations mixtes module les conclusions tirées jusqu'à présent concernant le rôle facilitateur de ces différents facteurs (dont deux ont trait au partage de l'information, et le troisième aux préférences initiales).

\subsection{Le rôle de l'expertise sur le partage de l'information en groupe}

Il est généralement admis que la présence d'experts influence le partage des informations uniques (pour une revue de question voir Stasser \& Titus, 2003). Un individu est considéré comme expert dans trois circonstances : s'il dispose de plus d'informations que les autres dans un domaine spécifique, s'il a davantage de compétences et enfin, s'il a plus d'expérience dans la tâche.

Une première série de recherches a mis en évidence l'effet positif de la connaissance par les membres du groupe de l'avantage informationnel de chacun dans un domaine donné. L'expertise provient ici de l'avantage informationnel qui est reconnu par les autres à l'intérieur du groupe. Dans ce cas, les groupes mentionnent plus d'informations uniques et découvrent plus fréquemment les profils cachés que les groupes où les experts ne sont pas reconnus (Stasser et al., 1995 ; Stasser, Vaughan, \& Stewart, 2000 ; Stewart \& Stasser, 1995). De plus, ils rappellent individuellement plus d'informations uniques après la discussion qu'en condition sans expertise reconnue, suggérant que les informations délivrées par les experts sont validées par les autres membres du groupe.

Une deuxième série de recherches a examiné l'effet du rôle d'expert en tant que membre du groupe qui dispose de compétences dans un domaine spécifique. Larson et al. (1996) ont montré que dans des équipes médicales, des internes répètent plus d'informations que des externes (des étudiants en médecine). Plus précisément, l'expert répète les informations communes en début de discussion, mais répète aussi les informations uniques au fur et à mesure que la discussion avance. En répétant ces informations, l'expert s'assure que l'attention du groupe reste focalisée sur les informations mentionnées et que celles-ci ne sont pas « oubliées » avec l'arrivée de nouvelles informations ${ }^{3}$.

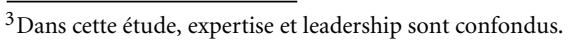


Enfin, une troisième série de recherches a analysé l'effet du rôle d'expert en tant que membre du groupe ayant le plus d'expérience dans une tâche donnée. Ainsi, Wittenbaum (1998, 2000), suggère que les membres ayant eu une expérience antérieure avec la tâche sont considérés comme des membres de haut statut dans le groupe. Elle montre que les membres de haut statut ne reproduisent pas le biais en faveur des informations communes dans la mesure où ils sont plus confiants quant aux informations qu'ils détiennent, ce qui n'est pas le cas des autres membres. Ce sentiment perçu de compétence permettrait aux experts de mentionner des informations uniques, et cela, même si elles ne peuvent pas être validées par les autres membres du groupe.

En somme, ces différentes recherches suggèrent que les experts améliorent le partage de l'information unique car ils sont capables de distinguer l'information pertinente de l'information non-pertinente (Shanteau, 1992). De même, ils savent récupérer l'information pertinente chez les autres membres (Abdolmohammadi \& Shanteau, 1992) et sont plus motivés à la répéter (Franz \& Larson, 2002). Nous suggérons que ces effets positifs identifiés dans la plupart de recherches sont typiques des interactions de groupe coopératives. Or, comme nous l'avançons tout au long de cet article, les situations de prise de décision en groupe impliquent pour ses membres des motivations mixtes (non seulement coopérer avec les autres mais aussi entrer en compétition avec eux) susceptibles de moduler l'effet de l'expertise sur le partage de l'information.

Une étude récente de Toma, Vasiljevic, Oberlé et Butera (2012) utilisant le profil caché - a examiné cette hypothèse. Les deux variables manipulées dans cette étude était l'assignation d'expertise et le contexte de coopération, compétition, tel qu'il a été manipulé par Toma et Butera (2009). Les résultats mettent en évidence qu'en coopération les groupes disposant d'experts assignés mentionnent et répètent plus d'informations uniques que les groupes sans experts assignés, alors qu'en compétition l'inverse est obtenu. De plus ce pattern a été reproduit pour la répétition des informations uniques. Le mécanisme responsable de cet effet est la capacité de reconnaitre à sa juste valeur la compétence des autres experts dans le groupe. En compétition les experts se sentent menacés par la compétence des autres et s'en défendent en s'attribuant plus de compétences qu'aux autres. En coopération au contraire, les experts reconnaissent que les autres membres du groupe sont au moins aussi compétents qu'eux-mêmes. Or, plus cette différence de compétence soi-autrui est importante, moins les membres du groupe partagent leurs informations uniques.

Cette recherche va donc clairement à l'encontre du résultat généralement admis dans la littérature selon laquelle l'expertise perçue est un 
facteur facilitant le partage d'informations (Stasser \& Titus, 2003 ; Wittenbaum et Stasser, 1996). Conformément à l'hypothèse générale développée dans cet article, les effets de l'expertise dépendent de la motivation des membres du groupe. Ainsi, l'expertise peut être bénéfique lorsque les membres du groupe travaillent dans un but de coopération, mais elle peut être délétère lorsque les membres du groupe ont des motivations compétitives. Aussi ne suffit-il pas d'assigner des rôles d'experts pour améliorer le partage de l'information. Encore faut-il s'assurer que la coopération prime dans les interactions entre les membres du groupe.

\subsection{Le rôle du type de tâche sur le partage de l'information en groupe}

La nature perçue de la tâche à résoudre est censée, elle aussi, influencer le partage des informations. Cette manipulation de la nature perçue de la tâche (par ex., Stasser \& Stewart, 1992) trouve son origine dans les travaux de Laughlin (Laughlin, 1980 ; Laughlin \& Ellis, 1986) proposant de catégoriser les différents types de tâches selon un continuum allant des tâches de jugement (c'est-à-dire, n'ayant pas de solution correcte objectivement démontrable) aux tâches intellectuelles (c'est-à-dire, ayant une seule solution correcte objectivement démontrable). Ainsi collectivement, les premières sont résolues grâce au consensus et à la validation sociale, alors que les secondes sont résolues grâce à l'application des règles normatives (logique, arithmétique, syntaxe, etc.) qui permettent de trouver la solution correcte.

Dans une tâche de type profil caché, Stasser et Stewart (1992) ont indiqué à la moitié des participants qu'il s'agissait d'une tâche de jugement et à l'autre moitié qu'il s'agissait d'une tâche intellectuelle. Dans ce dernier cas seulement, le partage des informations et la découverte du profil caché qui en résultait se sont avérés optimaux. L'objectif des groupes étant d'éviter de produire une solution erronée, leurs membres se sont investis davantage dans la recherche des informations critiques, c'est-à-dire les informations uniques. Ces résultats suggèrent que la perception de la nature de la tâche peut potentiellement affecter la gestion des ressources informationnelles des groupes. Cependant, ces résultats n'ont pas toujours été reproduits dans les études ultérieures (Campbell \& Stasser, 2006 ; Franz \& Larson, 2002 ; Hollingshead, 1996 ; Schittekatte, 1996 ; Stewart \& Stasser, 1998).

Poursuivant notre hypothèse générale, nous avançons que l'inconsistance de ces résultats est précisément due au fait que l'impact de 
la nature perçue de la tâche est également influencé par les motivations compétitives ou coopératives des membres du groupe. Toma (2007) a informé des groupes, placés soit en coopération soit en compétition, que la tâche qui leur a été confiée impliquait une seule vs. des multiples solutions (tâche intellectuelle vs. tâche de jugement). Les résultats révèlent que lorsque la tâche est perçue comme une tâche intellectuelle, les membres des groupes mentionnent plus d'informations uniques que d'informations communes, et cela indépendamment du contexte coopératif ou compétitif. En revanche, lorsque la tâche est définie comme une tâche de jugement, les membres des groupes mentionnent plus d'informations uniques que d'informations communes en coopération, alors que l'inverse est obtenu en compétition.

Rappelons que c'est précisément ce dernier résultat que montrent les recherches classiques dans le paradigme des profils cachés. Par conséquent, il est probable que dans ces recherches antérieures (voir Wittenbaum \& Stasser, 1996), les participants aient non seulement perçu les tâches utilisées comme des tâches de jugement, mais qu'ils aient également poursuivi des buts compétitifs. Evidemment, ce commentaire, qui restreint la généralité de résultats de Stasser, est provocateur et nécessitera un examen empirique approfondi. Aussi, les recherches futures devraient examiner la raison pour laquelle dans les tâches de jugement, plus que dans les tâches intellectuelles, les motivations mixtes ont un impact sur le partage de l'information. Une des explications suggérée par la littérature est que les tâches de jugement entrainent de l'incertitude, qui à son tour induit une tendance à la comparaison sociale et à la rétention de l'information.

\subsection{Le rôle des divergences des préférences initiales sur l'atténuation du biais de confirmation dans la décision de groupe}

Comme évoqué précédemment, l'un des mécanismes explicatifs des décisions collectives de piètre qualité est la difficulté qu'ont les membres du groupe à infirmer leurs préférences initiales. Justement, dans le profil caché, ces préférences sont souvent non seulement erronées, mais aussi partagées par tous les membres et cela avant même la discussion. Afin de contrecarrer leur effet néfaste (biais de confirmation), de nombreuses recherches manipulent l'orientation des membres du groupe vers une préférence initiale différente de façon à créer une divergence des solutions initiales (par ex., Brodbeck et al., 2002 ; Devine, 1999; Hightower \& Sayeed, 1996 ; Schulz-Hardt et al., 2006 ; Toma \& Butera 2009). Les résultats de ces 
recherches soulignent les bienfaits de la divergence des préférences initiales pour la discussion des informations uniques et la découverte consécutive du profil caché. En effet, confrontés au conflit des réponses, les membres du groupe sont moins confiants quant à la justesse de leur propre solution (Darnon, Harackiewicz, Butera, Mugny, \& Quiamzade, 2007 ; Sniezek \& Henry, 1989), génèrent plus de solutions alternatives (Crott, Giesel, \& Hoffmann, 1989) et sont moins egocentriques quant à leur solution initiale (Doise \& Mugny, 1984).

Néanmoins, les travaux de Mojzisch et Schulz-Hardt (2010) et ceux de Stasser et Titus (1985) suggèrent que la divergence d'opinions n'a pas toujours un effet positif. D'autres recherches encore - issues en particulier du domaine de l'influence sociale - le confirment. Par exemple, Nemeth et Rogers (1996), ainsi que Butera, Mugny, Legrenzi et Pérez (1996; voir aussi Butera \& Mugny, 2001) ont montré que si la source de divergence est une majorité ou un individu compétent, cela a comme effet une augmentation du biais de confirmation.

Il ressort de ces recherches que la divergence peut être bénéfique ou délétère, en fonction de sa signification sociale dans un contexte d'interaction. De la même manière, il est possible que le fait de percevoir les interactions avec les autres membres du groupe comme coopératives ou compétitives puisse jouer sur le fait de confirmer ou infirmer ses préférences initiales. Selon Deutsch (1973) la divergence avec les autres ne peut avoir des effets positifs que dans un contexte de coopération, car en coopération les individus font confiance aux autres, sont motivés à rechercher des solutions alternatives, expriment des opinions et informations variées et les prennent en considération de façon critique (Johnson \& Johnson, 1989, 1995, 2002).

Ainsi, dans un travail de groupe coopératif la divergence d'opinions devrait réduire la confirmation des préférences initiales et augmenter la qualité des décisions. En revanche, dans un contexte de compétition induisant la méfiance, la divergence d'opinions devrait induire des réactions défensives ayant comme conséquence une tendance à se focaliser sur la préférence initiale et à renforcer le biais de confirmation, avec des effets délétères sur la décision finale.

Cette hypothèse a été testée par Toma, Gilles, et Butera (2011). Dans leur étude, les membres du groupe étaient informés que les autres membres du groupe avaient des préférences initiales différentes (divergence) ou identiques (absence de divergence). Ils étaient confrontés à un complément d'informations sous forme de retranscription de la discussion (fictive) entre les deux autres membres de leur groupe avec lesquels ils s'attendaient à travailler de manière coopérative vs. compétitive. Tous les participants 
étaient invités à évaluer les informations reçues dans cette retranscription (dont la moitié était consistante et l'autre moitié inconsistante avec leur préférence initiale) et à prendre une décision finale en prenant en considération ce complément d'informations.

Conformément à l'hypothèse, les résultats ont mis en évidence qu'en coopération, la divergence conduit à une évaluation plus positive des informations inconsistantes et à des décisions plus appropriées. En compétition, elle amène une évaluation plus positive des informations consistantes (biais de confirmation), et nuit ainsi à la qualité de décision. En somme, cette recherche montre clairement que les effets positifs ou délétères de la divergence des préférences initiales sont fonction de la motivation à être coopératif ou compétitif. Les recherches futures devraient manipuler la divergence dans des tâches ayant une autre typologie et croiser les motivations sociales avec d'autres buts poursuivis par les groupes tels que la rapidité, la créativité etc.

\section{CONCLUSIONS ET PERSPECTIVES DE RECHERCHE}

Dans cet article, à la suite de Wittenbaum et al. (2004), et en nous inspirant du modèle du traitement motivé de l'information dans le groupe (De Dreu et al. 2007), nous avons remis en question le postulat selon lequel les membres des groupes qui prennent des décisions travaillent de manière coopérative. Nous avons avancé au contraire, que les membres d'un groupe sont guidés par des motivations mixtes, coopératives et compétitives, et nous avons étudié leur impact sur les préférences initiales des membres et les modalités du partage de l'information dans le cadre du paradigme des profils cachés.

Nous avons d'abord rappelé les résultats classiques obtenus dans le cadre de ce paradigme, résultats qui ont grandement enrichi les connaissances sur les décisions de groupe, mais qui restent entachés du soupçon d'être représentatifs d'un climat intellectuel où n'étaient envisagées dans les petits groupes que des interactions coopératives. On peut être étonné aujourd'hui par l'angélisme de cet a priori. Peut-être est-il la survivance de l'enthousiasme suscité dans les années 1930 par les études d'Elton Mayo, grâce auxquelles furent découvertes les potentialités intégratives du petit groupe informel comme lieu privilégié de relations harmonieuses et solidaires.

Ensuite, et c'est l'essentiel de notre propos, nous avons illustré à l'aide de résultats empiriques récents qu'une approche motivationnelle du 
traitement de l'information en groupe met clairement en lumière les effets différenciés sur les processus de la décision collective de consignes qui motivent à la coopération ou au contraire à la compétition.

La première conclusion qui s'impose, c'est que l'approche motivationnelle met très clairement en évidence la dimension stratégique du traitement de l'information. Ainsi, les études évoquées montrent que la compétition amène les membres du groupe à faire de la rétention de l'information unique, mais pas de l'information commune, ce qui n'est pas le cas dans les groupes coopératifs dans lesquels l'information unique est largement partagée (Toma et Butera, 2009). L'information unique étant seule susceptible de permettre la découverte de la solution optimale, on voit bien l'intérêt de sa rétention quand on veut être le premier à trouver la solution, et l'intérêt à la partager au plus vite quand le but est la réussite du groupe dans son ensemble. De la même manière, la confirmation des préférences initiales n'est pas non plus un biais systématique car c'est surtout en compétition que les membres du groupe sont focalisés vers leurs préférences initiales et évaluent de façon confirmatoire l'information partagée lors de la discussion. Dans les groupes coopératifs, au contraire, le partage de l'information unique sert à infirmer les préférences initiales (Toma \& Butera, 2009 ; Toma et al., 2011). Enfin, l'approche motivationnelle nuance également les résultats concernant les effets des facteurs classiquement conceptualisés comme facilitant l'optimisation des décisions de groupes, montrant même que ces facteurs peuvent se révéler être des entraves à la prise de décision optimale en groupe lorsque c'est la compétition qui est à l'œuvre. Ainsi, dans ce cas, la présence d'experts dans le groupe, reconnue habituellement comme un atout, n'est pas bénéfique, car eux, plus encore que des participants sans expertise, bloquent l'accès à l'information unique lorsqu'ils sentent à tort ou à raison leur compétence menacée (Toma et al., 2012). Il en va de même pour les bénéfices classiquement escomptés de la diversité des positions initiales. L'approche motivationnelle montre que dans des interactions compétitives, contrairement aux interactions coopératives, la diversité des positions initiales ne permet pas de surmonter le biais de confirmation, et n'aide donc pas à la découverte de la solution optimale (Toma et al., 2011).

L'ensemble de ces résultats montre de façon flagrante qu'ainsi appréhendé sous l'angle motivationnel, le partage sélectif de l'information n'est pas uniquement un biais probabiliste (Stasser \& Titus, 1985, 1987) ou un outil de validation sociale (Wittenbaum et al., 1999), mais clairement un comportement délibéré et stratégique qui sert les buts des membres du groupe (De Dreu et al., 2007) et a des conséquences sur la réussite de la 
tâche. Lorsque la structure de la tâche est basée sur une interdépendance des ressources et implique donc pour sa résolution optimale une mise en commun de celles-ci, la compétition a des effets délétères sur la décision finale.

Les travaux reportés ici sont donc cohérents avec le modèle de traitement motivé de l'information en groupe (De Dreu et al., 2008 ; Wittenbaum et al., 2005). Or, ce modèle, bien qu'offrant des perspectives théoriques très provocatrices, ne reçoit pas encore suffisamment de support empirique. L'approche de Wittenbaum et al. (2005) propose avant tout une alternative au paradigme du "profil caché », alors que l'approche de De Dreu et al. (2008) offre une perspective plus générale quant au rôle des différentes motivations sur la prise de décision en groupe. Selon ces auteurs, le partage et le traitement de l'information en groupe sont des processus intentionnels et stratégiques mis en place par les membres afin de satisfaire leurs buts. À leur tour, le partage et l'utilisation de l'information ont un impact sur la réussite de la tâche et la qualité des relations entre les membres du groupe (Figure 3 ). Ce modèle suggère que les processus motivationnels pourraient être étudiés en laboratoire moyennant

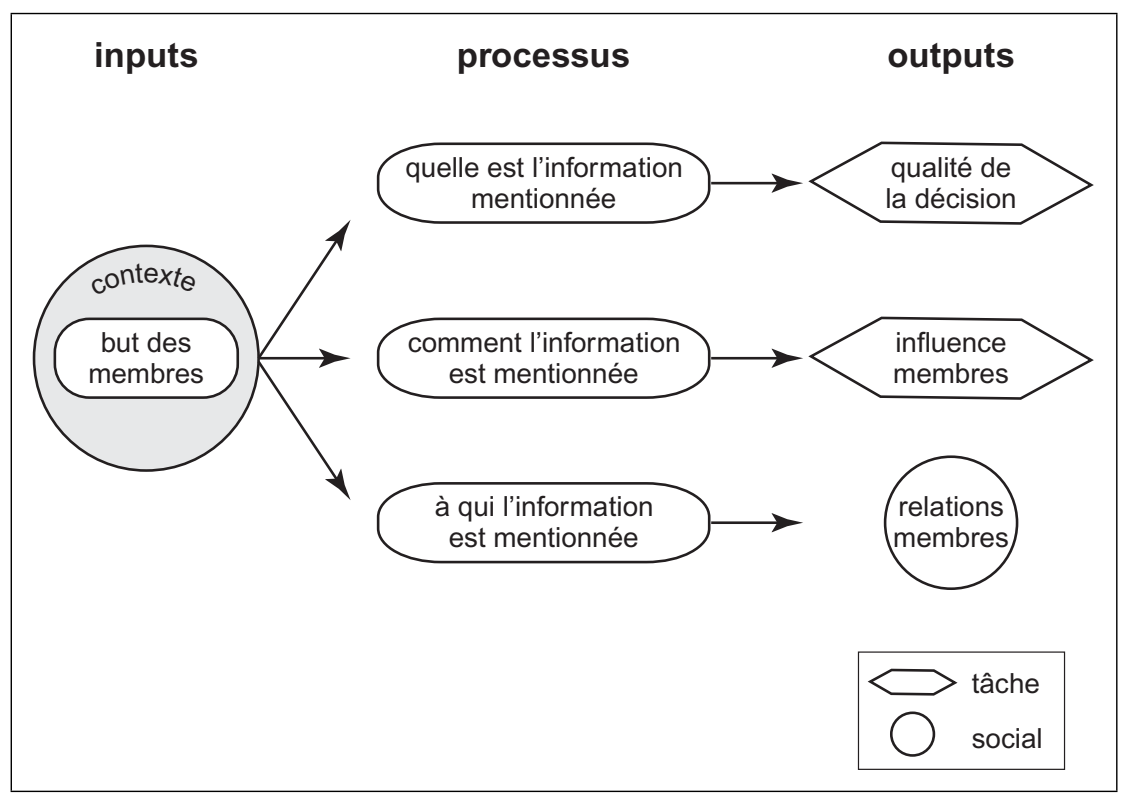

Figure 3. Le modèle de traitement motivé de l'information dans le groupe (selon Wittenbaum et al., 2004) 
des récompenses matérielles ou sociales, ou en induisant des pressions normatives ou temporelles à l'intérieur du groupe

La deuxième conclusion qui s'impose renvoie à l'intérêt de reconnaître l'existence de deux ordres de phénomènes dans les groupes de décision, l'un centré sur la tâche à effectuer, l'autre sur les membres du groupe (par ex., Oberlé \& Drozda-Senkowska, 2006). Si l'approche strictement opératoire des décisions en groupe a progressivement entraîné une focalisation sur la tâche au détriment d'une centration sur les membres, on voit bien que l'approche motivationnelle que nous défendons n'est possible que grâce à un intérêt renouvelé pour les processus inhérents aux membres du groupe. Cette approche motivationnelle implique surtout d'articuler les deux ordres de phénomènes pour cerner leur influence mutuelle sur les décisions. Ainsi, l'influence néfaste de la compétition n'est pas généralisable à la résolution de toutes les sortes de tâches possiblement confiées à un groupe. Un des résultats que nous présentons (Toma, 2007) réduit la généralité de ceux obtenus par Stasser et ses collaborateurs, en montrant que c'est seulement avec des tâches de jugement et lorsque les membres du groupe sont en compétition que l'on observe les résultats classiques de Stasser $(1985,1987)$.

Une caractéristique commune à toutes les études que nous avons présentées pour illustrer empiriquement l'intérêt de l'approche motivationnelle dans l'étude des décisions de groupe est qu'elles opposent la compétition et la coopération en tant que variables contextuelles censées affecter de la même manière tous les membres du groupe. Cette façon de procéder peut étonner pour deux raisons qu'il vaut la peine de discuter.

Premièrement cette procédure qui consiste à contraster les contextes coopératifs et compétitifs peut apparaître antinomique avec notre souci de mettre en évidence l'existence simultanée des motivations sociales mixtes, coopératives et compétitives (De Dreu et al., 2008). Dans des contextes réels de travail, il est rare de trouver des situations exclusivement et clairement coopératives ou compétitives. Malgré cette objection, la procédure choisie nous a semblé cependant un premier pas indispensable pour montrer que le partage des informations, l'infirmation des préférences initiales et les décisions prises n'étaient pas équivalentes en compétition et en coopération. La recherche future devra mettre sur pied des dispositifs expérimentaux qui permettront d'étudier l'impact de prescriptions mixtes - telles que "coopère et sois le meilleur ! ", injonction apparemment paradoxale, mais si fréquente en milieu professionnel et éducatif.

Deuxièmement, la littérature suggère que les individus diffèrent quant à leur orientation motivationnelle (par ex., McClintock \& Liebrand, 1988 ; Kelley \& Stahelski, 1970). Les recherches ont montré en particulier que les pro-sociaux ne maintiennent un comportement coopératif que 
lorsqu'ils travaillent avec d'autres pro-sociaux, alors que les compétiteurs maintiennent un comportement compétitif indépendamment du profil ou du comportement des autres (Miller \& Holmes, 1975 ; Van Lange, 1992). La recherche future devra prendre en compte la variabilité interindividuelle concernant la propension à être plus ou moins coopératif ou compétitif, ce qui nous paraît intéressant en particulier au regard de l'impact que la composition d'un groupe peut avoir sur le partage et l'utilisation de l'information en groupe en vue d'une décision.

Pour conclure cet article, il est utile d'alerter le lecteur sur un contresens qui ne doit pas être fait à sa lecture : ce travail n'avait pas pour ambition de faire de la coopération la panacée du traitement optimal de l'information en groupe, mais de mettre en lumière l'intérêt d'une approche motivationnelle de ce traitement. Dans ce but, nous nous sommes focalisés sur ce que De Dreu et al. (2008) appellent les motivations sociales. Cependant, ce ne sont pas les seules motivations à l'œuvre comme le montrent non seulement De Dreu et al. (2007) mais aussi les spécialistes du traitement dual de l'information (Chaiken et al., 1996 ; Chaiken, Liberman, \& Eagly, 1989) tout comme ceux qui défendent au contraire un modèle unidimensionnel (Kruglanski \& Webster, 1996 ; Kruglanski, Thompson, \& Spiegel, 1999). Tous relèvent en particulier l'existence d'une motivation épistémique qui renvoie à l'envie de connaissance en soi, d'où une exigence d'objectivité et d'exactitude. Tous insistent sur le fait que les différentes motivations peuvent coexister, se combiner entre elles, leurs effets pouvant s'ajouter ou s'annuler. Ainsi, le modèle de De Dreu et al. (2008) suggère que la coopération n'est bénéfique qu'à condition d'être associée à une motivation épistémique. À l'inverse, la motivation épistémique pourrait contrecarrer les effets néfastes de la compétition. Certaines études montrent effectivement que la compétition peut avoir des effets bénéfiques notamment sur la créativité (De Dreu \& Nijstad, 2008) et plus largement dans des tâches qui impliquent une pensée divergente (Beersma \& De Dreu, 2005).

En nous inscrivant dans la filiation des chercheurs qui étudient à quelles conditions non seulement sociocognitives mais aussi motivationnelles le travail en groupe est productif, nous espérons contribuer à surmonter l'impasse du débat sur la dimension réelle ou illusoire des bienfaits du groupe.

Reçu le 14 février 2011.

Révision acceptée le 6 décembre 2011. 


\section{BIBLIOGRAPHIE}

Abdolmohammadi, M. J., \& Shanteau, J. (1992). Personal attributes of expert auditors. Organizational Behavior and Human Decision Processes, 53, 158-172.

Augustinova, M. (2008). Falsification cueing in collective reasoning: Example of the Wason selection task. European Journal of Social Psychology, 38, 1-16.

Beersma, B., \& De Dreu, C. K. W. (2005). Conflict's consequences: Effects of social motives on post-negotiation creative and convergent group functioning and performance. Journal of Personality and Social Psychology, 89, 358-374.

Brauner, E., \& Scholl, W. (2000). Editorial: The information processing approach as a perspective for groups research. Group Processes \& Intergroup Relations, 3, 115-122. Brodbeck, F. C., Kerschreiter, R., Mojzisch, A., Frey, D., \& Schulz Hardt, S. (2002). The dissemination of critical, unshared information in decision-making groups: The effects of pre-discussion dissent. European Journal of Social Psychology, 32, 35-56.

Buchs, C., Butera, F., \& Mugny, G. (2004). Resource interdependence, student interactions and performance in cooperative learning. Educational Psychology, 24, 291314.

Buchs, C., Pulfrey, C., Gabarrot, F., \& Butera, F. (2010). Competitive conflict regulation and informational dependence in peer learning. European Journal of Social Psychology, 40, 418-435.

Burnstein, E., \& Vinokur, A. (1977). Persuasive argumentation and social comparison as determinants of attitude polarization. Journal of Experimental Social Psychology, 13, 315-332.

Butera, F., Darnon, C., Buchs, C. et Muller, D. (2006). Les méfaits de la compétition: Comparaison sociale et focalisation dans l'apprentissage. In R.V. Joule., \& P. Huguet
(Eds.), Bilans et perspectives en psychologie sociale. Grenoble: Presses Universitaires de Grenoble.

Butera, F., \& Mugny, G. (1995). Conflict between incompetences and influence of a low-competence source in hypothesis testing. European Journal of Social Psychology, 25, 457-462.

Butera, F., \& Mugny, G. (2001). Conflicts and social influences in hypothesis testing. Dans C. K. W. De Dreu, \& N. De Vries (Eds.), Group consensus and minority influence: implications for innovation. Oxford: Blackwell.

Butera, F., Mugny, G., Legrenzi, P., \& Pérez, J.A. (1996). Majority and minority influence, task representation, and inductive reasoning. British Journal of Social Psychology, 35, 123-136.

Campbell, J., \& Stasser, G. (2006). The influence of time and task demonstrability on decision-making in computer-mediated and face-to-face groups. Small Group $R e$ search, 37, 271-294.

Chaiken, S., Giner-Sorolla, R., \& Chen, S. (1996). Beyond accuracy: Defense and impression motives in heuristic and systematic information processing. In P. M. Gollwitzer, \& J. A. Bargh (Eds), The psychology of action: Linking cognition and motivation to behavior. New York, NY, US: Guilford Press

Chaiken, S., Liberman, A., \& Eagly, A. H. (1989). Heuristic and systematic information processing within and beyond the persuasion context. In J. S. Uleman, \& J. A. Bargh (Eds.), Unintended thought. New York, NY, US: Guilford Press.

Collins, B. E., \& Guetzkow, H. (1964). A social psychology of group processes for decision-making. New York, NY: Wiley.

Crott, H. W., Giesel, M., \& Hoffmann, C. (1998). The process of inductive inference 
in groups: the use of positive and negative hypothesis and target testing in sequential rule-discovery tasks. Journal of Personality and Social Psychology, 75, 938-952.

Darnon, C., Butera, F., \& Harackiewicz, J. (2007). Achievement goals in social interactions: Learning within a mastery vs. performance goal. Motivation and Emotion, 31, 61-70.

Darnon, C., Dompnier, B., Delmas, F., Pulfrey, C., \& Butera, F. (2009). Achievement goal promotion at university: Social desirability and social utility of mastery and performance goals. Journal of Personality and Social Psychology, 96, 119-134.

Darnon, C., Harackiewicz, J., Butera, F., Mugny, G., \& Quiamzade, A. (2007). Performance-approach and performanceavoidance goals: When uncertainty makes a difference. Personality and Social Psychology Bulletin, 33, 813-827.

Darnon, C., Muller, D., Schrager, S., Pannuzzo, N., \& Butera, F. (2006). Mastery and performance goals predict epistemic and relational conflict regulation. Journal of Educational Psychology 98, 766-776.

Davis, J. H. (1973). Group decision and social interaction: A theory of social decision schemes. Psychological Review, 80, 97-125.

Davis, J. H. (1996). Group decision making and quantitative judgments: A consensus model. In E. H. Witte \& J. H. Davis (Eds). Understanding group behavior: Consensual action by small groups. Mahwah, NJ: Erlbaum.

Davis, J. H., Laughlin, P. R., \& Komorita, S. S. (1976). The social psychology of small groups. Annual Review of Psychology, 27, 501-541.

De Dreu, C. K. W., Beersma, B., Stroebe, K., \& Euwema, M. C. (2006). Motivated information processing, strategic choice, and the quality of negotiated agreement. Journal of Personality and Social Psychology, 90, 927-943.

De Dreu, C. K. W., \& Carnevale, P. J. D. (2003). Motivational bases for information processing and strategic choice in conflict and negotiation. In M. P. Zanna (Ed.), Advances in Experimental Social Psychology. New York, NY, US: Academic Press.

De Dreu, C. K. W., \& McCusker, C. (1997). Gain-loss frame in two-person social dilemmas: A transformational analysis. Journal of Personality and Social Psychology, 72, 1093-1106.

De Dreu, C. K. W., \& Nijstad, B. A. (2008). Conflict and creativity: Threat-Rigidity or Motivated Focus? Journal of Personality and Social Psychology, 95, 648-661.

De Dreu, C. K. W., Nijstad, B. A., \& van Knippenberg, D. (2008). Motivated information processing in group judgment and decision making. Personality and Social Psychology Review, 12, 22-49.

De Dreu, C. K. W., \& Van Knippenberg, D. (2005). The possessive self as a barrier to constructive conflict management: Effects of mere ownership, process accountability, and self-concept clarity on competitive cognitions and behavior. Journal of Personality and Social Psychology, 89, 345-357.

Deutsch, M. (1973). The Resolution of conflict: Constructive and destructive processes. New Haven: Yale University Press.

Devine, D. J. (1999). Effects of cognitive ability, task knowledge, information sharing, and conflict on group decision-making effectiveness. Small Group Research, 30, 608-634.

Doise, W., \& Mugny, G. (1984). The social development of the intellect. Oxford, England: Pergamon Press.

Franz, T. M., \& Larson, J. R. (2002). The impact of experts on information sharing during group discussion. Small Group Research, 33, 383-411.

French, J. R., \& Raven, B. H. (1959). The bases of social power. In D. Cartwright (Ed.), Studies in social power. Ann Arbor, MI: Institute for Social Research.

Frey, D. (1986). Recent research on selective exposure to information. Advances in Experimental Social Psychology, 19, 41-80. 
Gigone, D., \& Hastie, R. (1993). The common knowledge effect: Information sharing and group judgment. Journal of Personality and Social Psychology, 65, 959-974.

Gigone, D., \& Hastie, R. (1997). The impact of information on small group choice. Journal of Personality and Social Psychology, 72, 132-140.

Greitemeyer, T., \& Schulz-Hardt, S. (2003). Preference-consistent evaluation of information in the hidden profile paradigm: Beyond group-level explanations for the dominance of shared information in group decisions. Journal of Personality and Social Psychology, 84, 322-339.

Hart, P.T. (1998). Preventing groupthink revisited: Evaluating and reforming groups in government. Organizational Behavior and Human Decision Processes, 73, 306-326.

Hastie, R. (1986). Review essay: Experimental evidence on accuracy. In G. Owen, \& B. Grofman (Eds.), Decision research. Greenwitch, CT: JAI Press.

Hastie, R., Penrod, S., \& Pennington, N. (1983). Inside the jury. Cambridge, MA, US: Harvard University Press.

Hightower, R., \& Sayeed, L. (1995). The impact of computer-mediated communication systems on biased group discussion. Computers in Human Behavior, 11, 33-44.

Hinsz, V. B., Tindale, R. S., \& Vollrath, D. A. (1997). The emerging conceptualization of groups as information processors. Psychological Bulletin, 121, 43-64.

Hollingshead, A. B. (1996). The rank-order effect in group decision making. Organizational Behavior and Human Decision Processes, 68, 181-193.

Janis, I. L. (1972). Victims of groupthink: A psychological study of foreign-policy decisions and fiascoes. Oxford, UK: Houghton Mifflin.

Janis, I.L., \& Mann, L. (1977). Decision making: A psychological analysis of conflict, choice, and commitment. New York, USA: Free Press.
Johnson, D. W., \& Johnson, R. (1989). Cooperation and competition: Theory and research. Edina, MN: Interaction Book Company.

Johnson, D. W., \& Johnson, R. (1995). Creative controversy: Intellectual challenge in the classroom. Minneapolis: Interaction Book Company.

Johnson, D. W., \& Johnson, R. (2002). Social interdependence theory and university instructions: theory into practice. Swiss Journal of Psychology, 61, 119-129.

Kaplan, M. F., \& Miller, C. E. (1987). Group decision making and normative versus informational influence: Effects of type of issue and assigned decision rule. Journal of Personality and Social Psychology, 53, 306-313.

Kasser, T., Cohn, S., Kanner, A. D., \& Ryan, R. M. (2007). Some costs of American corporate capitalism: A psychological exploration of value and goal conflicts. Psychological Inquiry, 18, 1-22.

Kelley, H. H., \& Stahelski, A. J. (1970). The inference of intention from moves in the Prisoner's Dilemma Game. Journal of Experimental Social Psychology, 6, 401-419.

Kelley, H. H., \& Thibaut, J. (1978). Interpersonal relations: A theory of interdependence, New York: Wiley.

Kerr, N. L., \& Tindale, R. S. (2004). Small group decision making and performance. Annual Review of Psychology, 55, 623-656.

Komorita, S. S., \& Parks, C. D. (1995). Interpersonal relations: Mixed-motive interaction. Annual Review of Psychology, 46, 183-207.

Kruglanski, A. W., Thompson, E. P., \& Spiegel, S. (1999). Separate or equal? Bimodal notions of persuasion and a singleprocess "unimodel". In S. Chaiken, \& Y. Trope (Eds.), Dual-process theories in social psychology. New York, NY, US: Guilford Press.

Kruglanski, A. W., \& Webster, D. M. (1996). Motivated closing of the mind: 'Seizing' 
and 'freezing'. Psychological Review, 103, 263-283.

Larson, J. R., Christensen, C., Abbott, A. S., \& Franz, T. M. (1996). Diagnosing groups: Charting the flow of information in medical decision-making teams. Journal of Personality and Social Psychology, 71, 315-330.

Larson, J. R., Foster-Fishman, P. G., \& Keys, C. B. (1994). Information sharing in decision-making groups. Journal of Personality and Social Psychology, 67, 446-461.

Larson, J. R., \& Harmon, V. M. (2007). Recalling shared vs. unshared information mentioned during group discussion: Toward understanding differential repetition rates. Group Processes and Intergroup Relations, 10, 311-322.

Laughlin, P. R. (1980). Social combination processes of cooperative problem-solving groups on verbal intellective tasks. In M. Fishbein (Ed.), Progress in social psychology. Hillsdale, NJ: Erlbaum.

Laughlin, P. R., \& Ellis, A. L. (1986). Demonstrability and social combination processes on mathematical intellective tasks. Journal of Experimental Social Psychology, 22, 177-189.

Lewicka, M. (1989). Toward a pragmatic perspective on cognition: Does evaluative meaning influence rationality of lay inferences? Polish Psychological Bulletin, 20, 267-285.

Lorge, I., \& Solomon, H. (1955). Two models of group behavior in the solution of Eureka-type problems. Psychometrika, 20, 139-148.

Mayo, E. (1945). The social problems of an industrial civilization. Oxford, UK: Graduate School of Business Adminis.

McClintock, C. G., \& Liebrand, W. B. (1988). Role of interdependence structure, individual value orientation, and another's strategy in social decision making: A transformational analysis. Journal of Personality and Social Psychology, 55, 396-409.
McGrath, J. E. (1984). Groups: Interaction and performance. Prentice Hall, Englewood Cliffs, NJ.

Miller, D. T., \& Holmes, J. G. (1975). The role of situational restrictiveness on selffulfilling prophecies: A theoretical and empirical extension of Kelley and Stahelski's triangle hypothesis. Journal of Personality and Social Psychology, 31, 661-673.

Moreland, R.L., \& Levine, J.M. (1992). The composition of small groups. Advances in Group Processes, 9, 237-280.

Mojzisch, A., \& Schulz-Hardt, S. (2010). Knowing others' preferences degrades the quality of group decisions. Journal of Personality and Social Psychology, 98, 794-808.

Nemeth, C. J., \& Rogers, J. (1996). Dissent and the search for information. British Journal of Social Psychology, 35, 67-76.

Oberlé, D., \& Drozda-Senkowska, E. (2006). Processus orientés vers la tâche vs processus orientés vers le groupe: Une vieille distinction toujours fructueuse? Cahiers Internationaux de Psychologie Sociale, 70, 63-72.

Parks, C. D., \& Cowlin, R. (1995). Group discussion as affected by number of alternatives and by a time limit. Organizational Behavior and Human Decision Processes, 62, 267-275.

Parks, C. D., \& Cowlin, R. A. (1996). Acceptance of uncommon information into group discussion when that information is or is not demonstable. Organizational Behavior and Human Decision Processes., 66, 307-315.

Postmes, T., Spears, R., \& Cihangir, S. (2001). Quality of decision making and group norms. Journal of Personality and Social Psychology, 80, 918-930.

Pruitt, D. G. (1998). Social conflict. In D. Gilbert, S. Fiske, \& G. Lindzey (Eds.), Handbook of social psychology. New York: McGraw-Hill.

Savadori, L., Van Swol, L. M., \& Sniezek, J. A. (2001). Information sampling and confidence within groups and judge advisor 
systems. Communication Research., 28, 737-771.

Schulz-Hardt, S., Brodbeck, F. C., Mojzisch, A., Kerschreiter, R., \& Frey, D. (2006). Group decision making in hidden profile situations: Dissent as a facilitator for decision quality. Journal of Personality and Social Psychology, 91, 1080-1093.

Shanteau, J. (1992). Competence in experts: The role of task characteristics. Organizational Behavior and Human Decision Processes, 53, 252-266.

Shaw, M.E. (1981). Group Dynamics: The Psychology of Small Group Behavior. McGraw-Hill, New York.

Schittekatte, M. (1996). Facilitating information exchange in small decision-making groups. European Journal of Social Psychology, 26, 537-556.

Schittekatte, M., \& Van Hiel, A. (1996). Effects of partially shared information and awareness of unshared information on information sampling. Small Group Research., 27, 431-449.

Sniezek, J. A., \& Henry, R. A. (1989). Accuracy and confidence in group judgment. Organizational Behavior and Human Decision Processes, 43, 1-28.

Stasser, G. (1999). The uncertain role of unshared information in collective choice. In L. L. Thompson, J. M. Levine, \& D. M. Messick (Eds.), Shared cognition in organizations: The management of knowledge. Mahwah, NJ, US: Lawrence Erlbaum Associates.

Stasser, G., \& Birchmeier, Z. (2003). Group creativity and collective choice. In P. Paulus, \& B. Nijstad (Eds.) Group Creativity. New York: Oxford University Press.

Stasser, G. et Davis, J. H. (1981). Group decision making and social influence: a social interaction model. Psychological Review, 88, 523-551.

Stasser, G., \& Dietz-Uhler, B. (2001). Collective choice, judgment, and problem solving. In M. A. Hogg, \& R. S. Tindale (Eds.) Blackwell handbook of social psychology: Group processes. Malden, MA: Blackwell Publishers.

Stasser, G., \& Stewart, D. (1992). Discovery of hidden profiles by decision-making groups: Solving a problem versus making a judgment. Journal of Personality and Social Psychology, 63, 426-434.

Stasser, G., Stewart, D. D., \& Wittenbaum, G. M. (1995). Expert roles and information exchange during discussion: The importance of knowing who knows what. Journal of Experimental Social Psychology, 31, 244-265.

Stasser, G., Taylor, L. A., \& Hanna, C. (1989). Information sampling in structured and unstructured discussions of three- and six-person groups. Journal of Personality and Social Psychology, 57, 67-78.

Stasser, G., \& Titus, W. (1985). Pooling of unshared information in group decision making: Biased information sampling during discussion. Journal of Personality and Social Psychology, 48, 1467-1478.

Stasser, G., \& Titus, W. (1987). Effects of information load and percentage of shared information on the dissemination of unshared information during group discussion. Journal of Personality and Social Psychology, 53, 81-93.

Stasser, G., \& Titus, W. (2003). Hidden profiles: A brief history. Psychological Inquiry, 14, 304-313.

Stasser, G., Vaughan, S. I., \& Stewart, D. D. (2000). Pooling unshared information: The benefits of knowing how access to information is distributed among group members. Organizational Behavior and Human Decision Processes, 82, 102-116.

Steinel, W., \& De Dreu, C. K. W. (2004). Social motives and strategic misrepresentation in social decision making. Journal of Personality and Social Psychology, 86, 419-434.

Steiner, I. D. (1972). Group process and productivity. New York: Academic Press. 
Stewart, D. D., \& Stasser, G. (1995). Expert role assignment and information sampling during collective recall and decision making. Journal of Personality and Social Psychology, 69, 619-628.

Stewart, D. D., \& Stasser, G. (1998). The sampling of critical, unshared information in decision-making groups: The role of an informed minority. European Journal of Social Psychology, 28, 95-113.

Tindale, R. S., \& Kameda, T. (2000). "Social sharedness" as a unifying theme for information processing in groups. Group Processes and Intergroup Relations, 3, 123-140.

Tindale, R. S., Meisenhelder, H. M., Dykema-Engblade, A. A., \& Hogg, M. A. (2001). Shared cognitions in small groups. In M. A. Hogg, \& R. S. Tindale (Eds.), Blackwell handbook in social psychology: Group processes. Oxford, UK: Blackwell Publishers.

Tindale, R. S., \& Sheffey, S. (2002). Shared information, cognitive load, and group memory. Group Processes and Intergroup Relations, 5, 5-18.

Toma, C. (2007). Strategic information sharing and use in group decision making: the role of cooperation and competition. Thèse de doctorat, Université Grenoble 2.

Toma, C., \& Butera, F. (2009). Hidden profiles and concealed information: Strategic information sharing in group decision making. Personality and Social Psychology Bulletin, 35, 793-806.

Toma, C., Gilles, I., \& Butera, F. (2011). Strategic use of preference confirmation in group decision making: the role of competition and dissent. British Journal of Social Psychology.

Toma, C., Vasiljevic, D., Oberlé, D., \& Butera, F. (2012). Assigned experts with competitive goals withhold information in group decision making. British Journal of Social Psychology.
Tjosvold., D. (1986). Dynamics of interdependence in organizations. Human Relations. 39. 517-540.

Tjosvold, D. (1990). Cooperation and competition in restructuring an organization. Canadian Journal of Administrative Sciences, 7, 48-54.

Van Lange, P. A. M. (1992). Rationality and morality in social dilemmas: The influence of social value orientations. In W. B. G. Liebrand, D. M. Messick, \& H. A. M. Wilke (Eds.), Social dilemmas: Theoretical issues and research findings. London: Pergamon Press.

Webster, D. M., \& Kruglanski, A. W. (1998). Cognitive and social consequences of the need for cognitive closure. In W. Stroebe, \& M. Hewstone (Eds.), European Review of Social Psychology. Hoboken, NJ, US: John Wiley \& Sons Inc.

Winquist, J. R., \& Larson, J. R. (1998). Information pooling: When it impacts group decision making. Journal of Personality and Social Psychology, 74, 371-377.

Wittenbaum, G. M. (1998). Information sampling in decision-making groups: The impact of members' task-relevant status. Small Group Research, 29, 57-84.

Wittenbaum, G. M. (2000). The bias toward discussing shared information. Why are high-status group members immune? Communication Research, 27, 379-401.

Wittenbaum, G. M., Hollingshead, A. B., \& Botero, I. C. (2004). From cooperative to motivated information sharing in groups: moving beyond the hidden profile paradigm. Communication Monographs, 71, 286-310.

Wittenbaum, G. M., \& Stasser, G. (1996). Management of information in small groups. In. A. M. Brower, \& J. L. Nye (Eds.), What's social about social cognition? Research on socially shared cognition in small groups. Thousand Oaks, CA, US: Sage Publications. 